\title{
MACHINE LEARNING CONTENT ADAPTIVE FILTERS FOR IMAGE DE-BLURRING
}

\author{
Pradip Mainali \\ OneSpan, Belgium ${ }^{1}$
}

\begin{abstract}
This paper proposes a novel algorithm to recover blindly a sharp image from its degraded form by pixel pattern classification and filtering by using the deconvolution filters trained for the corresponding pixel pattern. Noise amplification is a well-known phenomenon that arises due to an ill-posed nature of the deconvolution, which is tackled better with the pattern classification, thereby state-of-the-art image de-blurring quality is demonstrated, yet at the very low complexity. For the computational efficiency, the pixel pattern classes are learnt in a multi-layer structure consisting of the coarser and the finer patterns, reducing the pattern search complexity in the dictionary by a factor of 6 without losing any quality. As compared to deep-learning based methods, the proposed approach provides a smaller memory footprint and lightweight implementation. For lightweight and real-time implementation, the paper also validates a simple class matching metric of $l_{1}$-norm. The proposed method is suitable for embedded applications such as camera, TV systems, etc.
\end{abstract}

\section{KEYWORDS}

De-blurring, Deconvolution, Restoration

\section{INTRODUCTION}

Images are degraded with blur and noise during an image formation process for a number of reasons. The degradation process is expressed as:

$$
\mathbf{I}_{m}=\mathbf{I}_{a} \otimes \mathbf{h}+\eta
$$

where $\mathbf{I}_{m}$ is a measured blurry and noisy version of an actual image $\mathbf{I}_{a}, \mathbf{h}$ is a PSF (Point Spread Function) or blurring kernel and $\eta$ is the noise of the camera system. To recover an actual image from the measured image, a deconvolution/inverse filtering of the measured image is required with the filter $\mathbf{h}$. The inverse filtering is in general an ill-posed problem in presence of the noise (Bertero, 1998), hence some regularization is required (Bertero, 1998; Hansen, 1998; Vogel, 2002). Regularization is studied extensively to alleviate this problem and the proposed scheme provides regularization with training.

This paper proposes a low-cost algorithm to recover blindly a sharp image from a single blurry image based on the pixel pattern classification as shown in Figure 1. The pixel $p_{1}$ shown in Figure 1 is recovered by extracting the pixel feature by using the pixels in the $n \times n$ aperture in the blurry image. Subsequently, the $m$ nearest matching pixel patterns are searched in the dictionary and then the pixel is filtered with the filters trained for the corresponding pixel classes. Finally, the responses from the filters are combined by weighting with their class similarity distances. The noise contribution in the restored image can be reduced, if the deconvolution filters are adapted to the local SNR (signal-to-noise-ratio) of the pixel regions. For example, the low SNR region such as flat pattern $p_{0}$ shown in Figure 1 needs less enhancement to avoid noise amplification whereas the higher SNR region such as edge pattern $p_{1}$ can be enhanced more.

This paper reduces the computational complexity of the method based on pixel classification proposed in (Mainali, 2015) by using multi-layer pixel structure in the dictionary as shown in the right side of Figure 1, reducing significantly the class search complexity in the dictionary. With the two layer class structure

\footnotetext{
1 This work was carried out while the author was working at TPVision, Belgium.
} 
consisting of the $r=96$ coarse classes and each coarser classes clustered into the $f=8$ finer classes for the $5 \times 5$ aperture, achieves 6 times less complexity as compared to the algorithm proposed in (Mainali, 2015) having the single layer of the 768 pixel classes in the dictionary. Second, matching the pixel class in the dictionary is also a complex operation depending on the similarity metric that is used. In this paper, $l_{1}$-norm is selected as the similarity metric as compared to $l_{2}$-norm in (Mainali, 2015). The experimental results also show that no noticeable visual loss on the image sharpness was observed. The main advantage of the proposed approach as compared to the deep convolutional neural network based approaches (Schuler, 2013; $\mathrm{Xu}$, 2014; Hradi, 2015; Kupyn, 2017; Nah, 2017) is that the proposed method has smaller memory footprint of the parameters and the better computational efficiency, requiring no multiplier in searching pixel class in the dictionary. Therefore, opening up a lightweight implementation of the algorithm in the FPGA device for a real-time video processing for the application such as TV, camera systems etc. The proposed scheme is mainly suitable to remove the optical blur in the image, while the methods based on the deep convolutional neural network based approaches can be used to remove many different types of artifacts such optical blur, motion blur, etc.
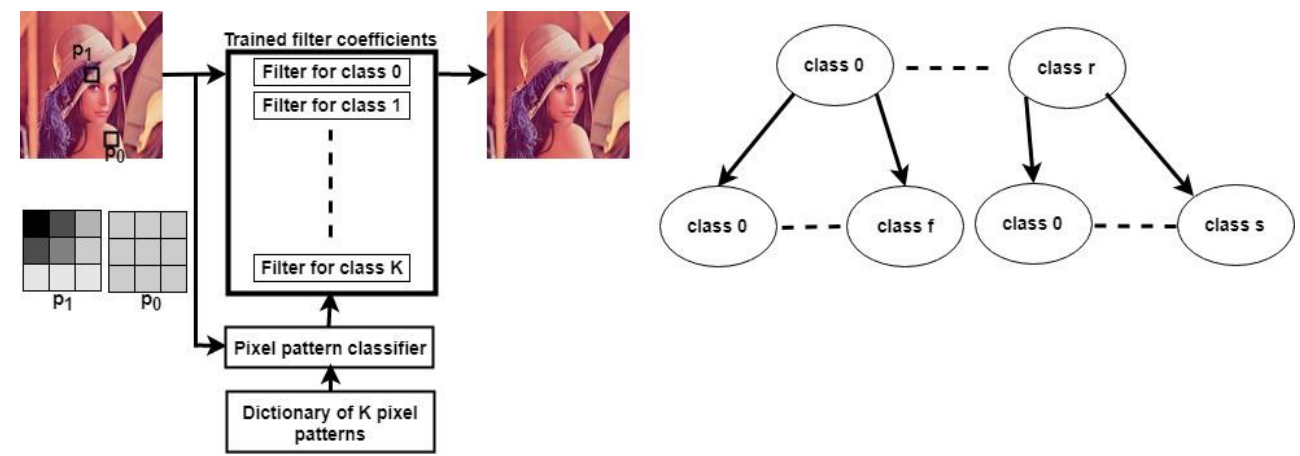

Figure 1. Recovering a sharp image with the pixel pattern classification and the trained filters (left) and multi-layered pixel patterns with the coarser and finer patterns (right)

The paper organization is as follows. Sec. 2 provides a quick survey of the image de-blurring algorithms and comparison with the proposed approach. The proposed image de-blurring algorithm is explained Sec. 3. The experimental results are given in Sec. 4 . Sec. 5 concludes the paper.

\section{RELATED WORK}

In the past, several image de-blurring algorithms are proposed. The methods can be broadly categorized into four different categories. These methods in the first category proposed in (Bilcu, 2008; Horiuchi, 2007; Nam, 2012) inject a high frequency details derived from the original image by filtering it with the derivative filter. Historically, this category of the methods appeared as the first step in developing the deblurring algorithms. These methods are still very widely used in many applications such as in the TV systems, photo-shop, camera etc. due to their low complexity and lightweight implementation for real-time applications. However, the same filter is used to filter every pixel, which enhances only the frequency details that are inside the frequency spectrum of the filter and also leads to the amplification of the noise in the flat region.

The methods in the second category are based on the iterative approach with some priors. Most of the methods in this category also assumes that a PSF is known. The methods based on probabilistic image priors are proposed in (Levin, 2007; Joshi, 2009; Krishnan, 2009; Levin, 2009; Schmidt, 2011; Zoran, 2011; Xu, 2014). They seek a maximum posteriori (MAP) estimate of the clean image, given a blurry (and noisy) version and a PSF. The iterative approach for the deconvolution by using the sparsity constraint in the wavelet transform domain were proposed in (Daubechies, 2004; Figueiredo, 2003; Vonesch, 2008). However, due to an iterative nature of the algorithm, the methods in this category are generally not suitable for the real-time implementations. 
The methods in the third category are based on the two step approach. First step is an inverse filtering in the frequency domain followed by the second step of denoising (Foi, 2006; Dabov, 2008; Schuler, 2013). Most of the methods in this category also assumes that a PSF is known. These methods apply a regularized inversion of the blur with a known blur PSF in the Fourier domain. This results in the sharper image, but it also has an effect of the noise amplification. The method in (Schuler, 2013) uses a neural network to clean the noise.

The methods in the fourth category are based on the machine learning approach. The image de-blurring algorithms based on machine learning using a deep convolutional neural network were proposed in (Xu, 2014; Hradi, 2015; Kupyn, 2017; Nah, 2017). The methods based on the pixel pattern classification were proposed in (Kondo, 2002; Ling, 2008), using handcrafted binary coded pixel classes. The main contributions of this paper compared to the previous works based on the binary coded pixel classes is that the proposed method automatically learns the pixel pattern classes from the images in the database instead of hand-crafting them.

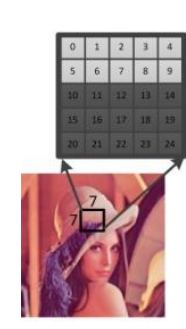

(a)

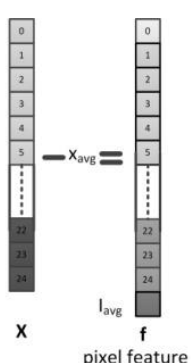

pixel feature

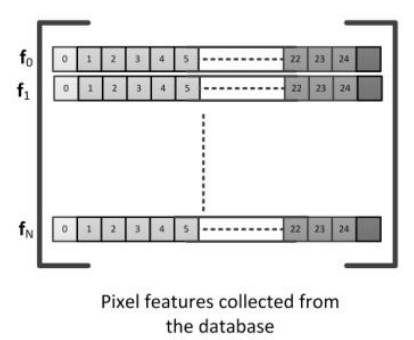

(b)

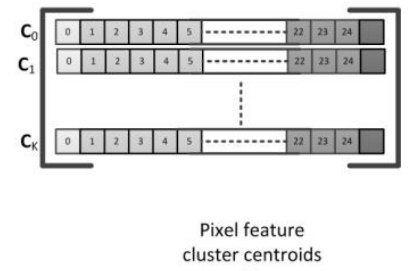

(c)

Figure 2. (a) Pixel feature extraction from the aperture of size $5 \times 5$. (b) Feature matrix of all the pixels in the database. (c) Learnt dictionary of pixel feature vectors (cluster centroids)

\section{PROPOSED ALGORITHM}

The blur phenomenon during the image acquisition is often modelled by the forward Gaussian convolution (Horiuchi, 2007; Joshi, 2009) and noise with an additive white Gaussian noise. The images in the training database consists of various scene types such as the indoor, outdoor, natural, dark/bright images etc. The degraded images are computed by applying the Gaussian blur and additive white Gaussian noise (AWGN) for the images in the training database. In the training database, there are $8 \times 10^{9}$ number of the pixels for the training. The training is performed for various blur $\left(\sigma_{b}=[0.8,1.1,1.4, \ldots, 5.0]\right)$ and noise settings $\left(\sigma_{n}=[0.0,1.0,2.0, \ldots, 5.0]\right)$. The offline learning and on-the-fly filtering stages are explained in Secs. 3.1 and 3.2 respectively.

\subsection{Offline Learning}

In the offline training, the algorithm learns the dictionary of pixel classes and the corresponding deconvolution filter kernels. The pixel feature consisting of the dynamic information and the average value is extracted by using the pixels within an aperture of size $n \times n$ as shown in Figure 2(a) as in (Mainali 2015), with the dynamic information computed as:

$$
\mathbf{f}=\mathbf{X}-x_{\text {avg }}
$$

where $\mathbf{X}$ and $x_{\text {avg }}$ are the pixels within the aperture and the average value of the pixels respectively. This type of pixel feature is suggested because after many experiments it was observed that it works very well for the sharpness enhancement as compared to the other features such as using pixel values, gradients etc. It is also simple to compute. The feature vector captures varying (i.e. ac) and fixed (i.e. dc) part of the pixel. Theoretically, for the sharpness enhancement, the varying part needs to be modified and hence is well suited for the sharpness enhancement. 
To learn the dictionary, the clustering algorithm is used to cluster the pixel feature vectors as shown in Figure 2(b). For the computational efficiency, the dictionary of the pixel patterns is learnt in a multi-layer structure consisting of the coarser and finer pixel classes. For our application for TV, the two layered network is used to de-blur image/video. The single layer of pixel classes with $K=768$ classes for the $5 \times 5$ aperture size was used in (Mainali 2015). For the two layer pixel classes, a solution consisting of the 96 coarser pixel classes with each coarse pixel class divided over 8 finer pixel classes was observed to be suitable for the de-blurring application. Once the dictionary is learnt, the next step is training the filters for each pixel pattern for the different blur and noise model parameters. The filters are trained only for the finer pixel pattern classes, as the filtering of a pixel with the coarser filters are not yet explored.

To train the filter $h_{c}$ for each class $c$, the least mean square error optimization cost function between the source pixels $\left(x_{s}\right)$ and the degraded pixels $\left(x_{d}\right)$ is used:

$$
e_{c}^{2}=\sum_{j=1}^{N_{c}}\left(x_{s}-\sum_{i \in(n \times n)} h_{c}(i) x_{d}(j, i)\right)^{2}
$$

where $N_{c}$ is the total number of the pixels that belongs to the class $c$. The degraded image is generated as: $I_{\mathrm{d}}$ $=I_{\mathrm{s}} \otimes G+\eta$, where $G$ is the Gaussian blurring kernel of standard deviation $\sigma_{b}$ and $\eta$ is Gaussian noise of standard deviation $\sigma_{n}$ and zero mean.

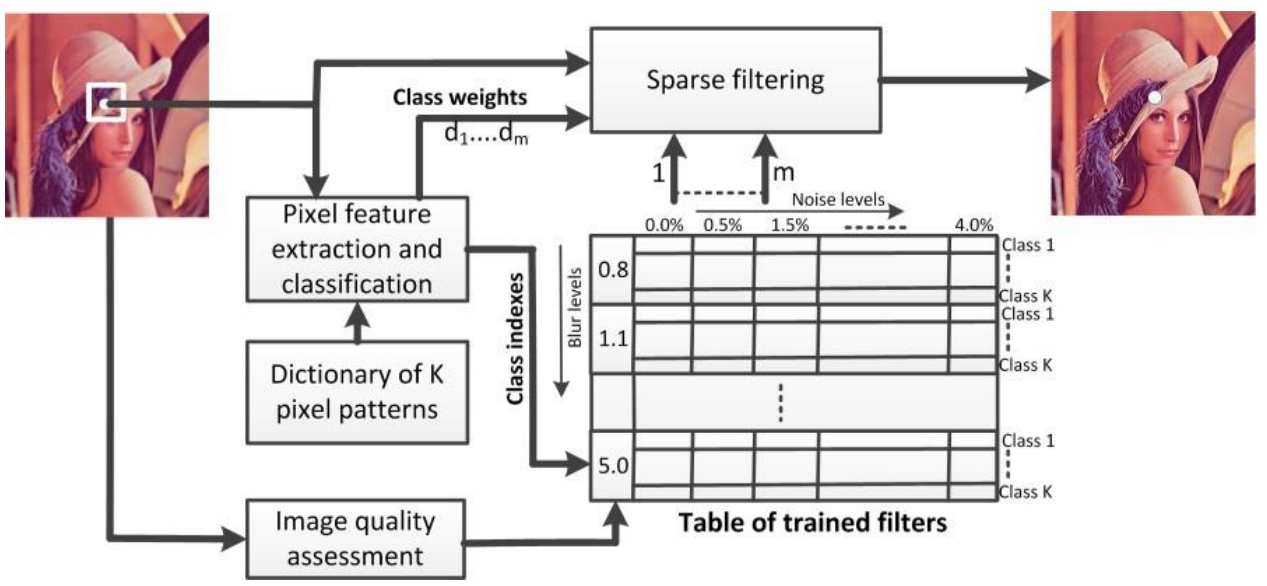

Figure 3. Recovering the image sharpness with the image quality estimation, the pixel pattern classification and the pattern adaptive trained filters

The optimal values of the filter coefficients for the class (c) is estimated by taking the first derivative of $e_{c}^{2}$ and by setting it to zero. After simplification, the following linear equation is solved to estimate the filter coefficients $\left(\mathbf{h}_{c}\right)$ for each class (c) (Mainali 2015):

$$
\mathbf{A}_{c} \mathbf{h}_{c}=\mathbf{b}_{c}
$$

where,

$$
\mathbf{A}_{c}=\sum_{j=1}^{N_{c}} \mathbf{X}_{d, j} \mathbf{X}_{d, j}^{T}, \quad b_{c}=\sum_{j=1}^{N_{c}} x_{s, j} \mathbf{X}_{d, j}
$$

where $\mathbf{X}_{d, j}$ is the lexicographically arranged pixels in the aperture as the column vector from the degraded image, $x_{s, j}$ is the $j^{\text {th }}$ pixel from the source image and $N_{c}$ is the total number of pixels belonging to the class $c$. The least square solution for the filter coefficients $\left(\mathbf{h}_{c}\right)$ for the class $\mathrm{c}$ is computed by using SVD. The training process is repeated for the different Gaussian blur $\left(\sigma_{b}=[0.8,1.1,1.4, \ldots 5.0]\right)$ and noise $\left(\sigma_{n}=[0.0\right.$, $1.0,2.0, \ldots 5.0])$ values. So, in total $6 \mathrm{MB}$ of data including dictionary and trained filters needs to be stored, with the data values stored in the 16-bit precision format.

\subsection{On-the-fly Filtering}

Figure 3 shows the block diagram for filtering the image. The algorithm consists of image quality assessment, the pixel feature extraction and classification blocks. The image quality assessment block is used to select the correct filter set (i.e. $\left(\sigma_{b}, \sigma_{n}\right)$ used in training) that is most suitable for the input image. 
To measure the blur and noise impacts present in the input image, the cumulative histogram of pixel transition delta (i.e. difference between maximum and minimum value within the $3 \times 3$ aperture) value is computed over the whole image. The transition delta values at 0.98 and 0.02 in the cumulative histogram are used to select the filter set. The former value (i.e. fluctuations of larger values) indicates the sharpness and the later one indicates the noise (i.e. fluctuations of small values) in an image. Once the global blur and noise impacts present in the input image is measured, the filter set trained with the nearest blur and noise model parameters is selected. The former value indicates that the $98 \%$ pixels in the image have transition delta below this value. The higher value indicates the sharper picture whereas the lower value indicates the blurry image. The experiment is also carried out to check how this value is changed due to the blur impact. Figure 4a shows the cumulative histogram of the transition delta values for the images blurred with the Gaussian blur values of $\sigma=1.0,2.0,3.0$. The transition delta values at 0.98 cumulative histogram decreases with the increasing blur values. Figure $4 \mathrm{~b}$ shows the cumulative histogram of the transition delta values for the image by adding the AWGN for the noise levels of $\sigma: 2.0,3.0,5.0$. The transition delta values at 0.02 cumulative histogram increases, indicating that the flat and texture regions in the image are corrupted more with the noise. The appropriate setting for the noise model parameters are derived by using the translation table from the measured values. It is also interesting to note that the measurement of the blur impact (i.e. values at 0.98) is less influenced with the addition of noise.

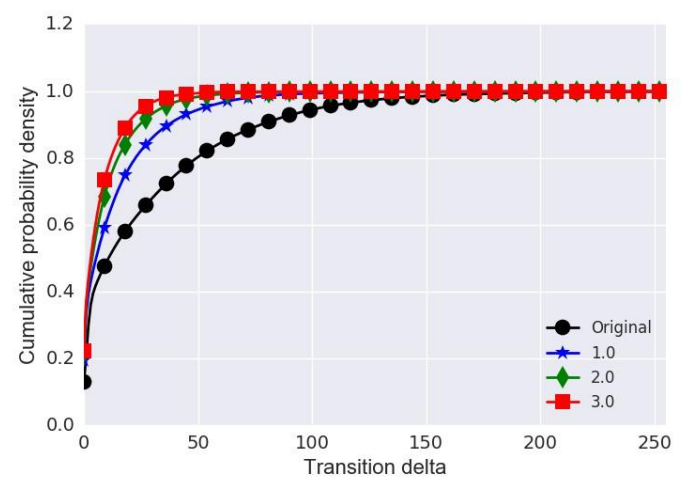

(a)

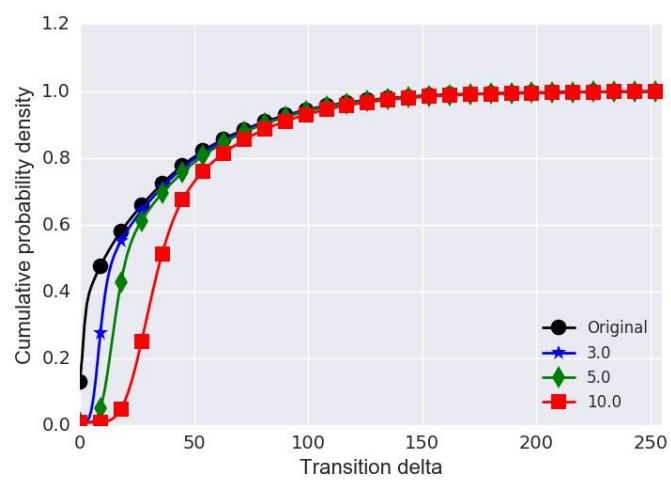

(b)

Figure 4. (a) Cumulative histogram for the Gaussian blur 1.0, 2.0 and 3.0. (b) Cumulative histogram for noise of 2.0, 3.0 and 5.0

The pixel feature vector is computed exactly as during the training stage and the nearest matching pixel feature patterns are searched in the dictionary. As the dictionary is organized in the coarser and finer pixel patterns, the $m$ nearest matching coarse pixel classes are searched. In this paper, $m=4$ is chosen. Once the coarse pixel classes are selected, the class distances are computed with all the finer pixel classes belonging to these $m$ coarser pixel classes. The class distances are normalized. The filters corresponding to the finer pixel classes are used to filter the pixel. The filtering results from these multiple filters are combined by weighting the filtered results with the class similarity distances $\left(d_{c}\right)$.

In this paper, $l_{1}$-norm is used as a similarity metric to find the nearest matching coarse pixel classes as compared to $l_{2}$-norm in (Mainali, 2015), which requires only the subtract and add operations, hence eliminates the complex and scarce multiplier operations in the FPGA device. Figure 5 shows the visual comparison of the results obtained with $l_{2}$-norm and $l_{1}$-norm search technique, where no visible loss on the perceived image quality was observed.

To filter image the algorithm only needs to load 768 different pixel classes, hence the method requires a very small footprint while the methods based on deep convolutional neural network has very high memory footprint requiring to load millions of parameters. Also, filtering is needed to perform with only small set of 32 filters, hence requiring a very low computational cost. 

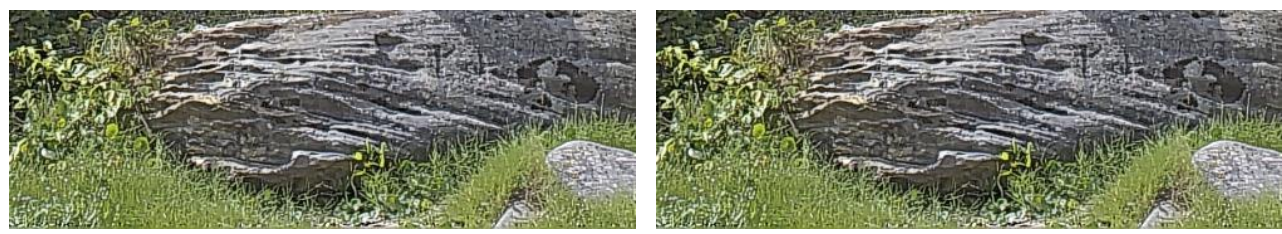

Figure 5. The image sharpness restored with (left) $l_{2}$ and (right) $l_{1}$ techniques

\section{EXPERIMENTAL RESULTS}

In this section, the experimental results are given to compare the quality of the image sharpness recovered by the state-of-art methods: BM3D (Dabov, 2008), the peaking approach (Rieder, 2001) and the method proposed in (Mainali 2015) using a single layer of pixel classes. The peaking approach is widely used in the TV system, photo-shop etc. and hence included in the comparison. The computational complexity is also compared with the single class layer approach in (Mainali 2015). For the BM3D algorithm, the default settings of the alpha sharp $=1.4$ and the noise $\sigma=1.0$ are used. The algorithm proposed in (Mainali, 2015) is configured with $K=768$ pixel classes for the $5 \times 5$ aperture size and $m=32$. The proposed algorithm is configured with the two layers of the pixel classes consisting of the 96 coarser pixel classes and the 8 finer pixel classes for each coarser pixel class, $m=4$ and the aperture of size $5 \times 5$ is used. The blur meter proposed in (Crete, 2007) is used to measure quantitatively the sharpness of the original and recovered images. The blur measurement results are provided in the range $[0,1]$, with a lower value indicating the higher sharpness.

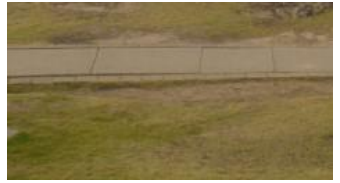

(a)

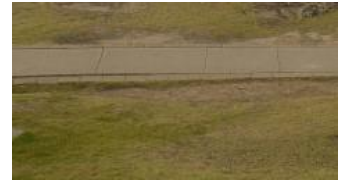

(b)

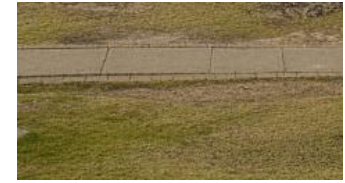

(c)

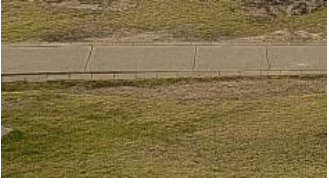

(d)

Figure 6. (a) The original blurry image with blur index 0.36 . The image sharpness restored with: (b) peaking with blur index 0.21 (c) BM3D with blur index 0.19 and (d) the proposed method with blur index 0.14

Table 1 gives the mean and the variance of the blur index values of the original and sharper images that were recovered by the proposed algorithm, BM3D, the peaking approach and the method given in (Mainali, 2015), evaluated over 50 different real-life images. These test images were not present in the training database. Table shows that the proposed method achieves the lowest blur index values, indicating the more sharply recovered images.

Figures 6 and 7 show visual comparison for the two different test images, the small extracted portion of the original blurry image and the images after the sharpness restoration by BM3D, the peaking and the proposed algorithm. The blur measurements are 32, 44, 63 and 55 respectively. The noise measurements are: $0,0,4,9$ respectively. With these measurements from the quality assessment block, the algorithm selects the filter set of pairs trained with $\left(\sigma_{b}, \sigma_{n}\right)$ of $(3.5,0)$ and $(2.3,2.0)$ respectively for these images. The visual results in the figures show that the proposed method generates visually sharper images. It is also observed that the proposed method recovers the finer details more sharply in the grass region in Figure 6 and also without noticeably enhancing the noise. On the other-hand, noise is amplified in the stone region by BM3D. Finally, for the challenging image in Figure 7 consisting of significant amount of noise, the proposed method enhances very little noise whereas the BM3D algorithm enhances the noise much more. For example, in the sky and the red tower of the bridge regions in Figure 7, the noise is enhanced much less by proposed algorithm as compared to the BM3D algorithm.

Table 2 gives the execution time comparison with the method in (Mainali, 2015) for the aperture sizes $3 \times 3$ and $5 \times 5$ on an Intel Xeon $2.1 \mathrm{GHz}$ CPU with 4GB RAM for the UHD image of the size $3840 \times 2160$. The proposed method in this paper achieve 3 and 6 times less complexity respectively as compared to approach in (Mainali, 2015), as the complexity in searching the nearest matching pixel class in 
the dictionary is reduced significantly. The method proposed in this paper consists of the 32 coarser, the 4 finer classes, the 96 coarser, and the 8 finer pixel classes for the former and the later aperture sizes. The method proposed in (Mainali, 2015) consists of the 128 and 768 pixel classes respectively for these two aperture sizes.

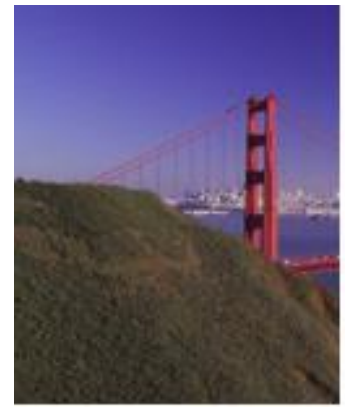

(a)

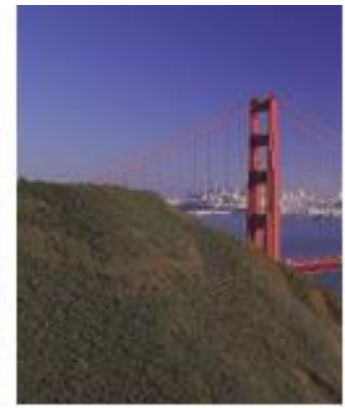

(b)

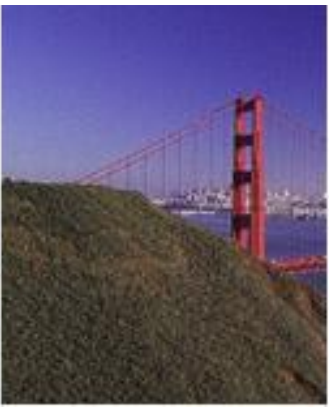

(c)

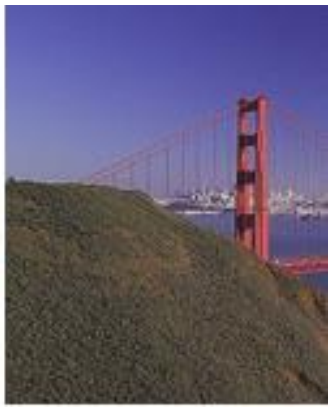

(d)

Figure 7. (a) The original blurry image with blur index 0.18 . The image sharpness restored with: (b) peaking with blur index 0.17 (c) BM3D with blur index 0.15 and (d) the proposed algorithm with blur index 0.12

Table 1. Blur index before and after sharpness restoration

\begin{tabular}{c|c|c|c}
\hline Original & Umask & BM3D & Proposed \\
\hline $0.38 \pm 0.16$ & $0.22 \pm 0.019$ & $0.19 \pm 0.020$ & $0.13 \pm 0.018$
\end{tabular}

Table 2. Execution time (secs.) for $3840 \times 2160$ image size

\begin{tabular}{c|c|c}
\hline & $3 \times 3$ & $5 \times 5$ \\
\hline Proposed algorithm & 1.70 & 6.80 \\
\hline (Mainali, 2015) & 5.62 & 43.84 \\
\hline
\end{tabular}

\section{CONCLUSION}

The paper proposed an image de-blurring algorithm based on the pixel pattern classification. To sharpen the image with different amount of blur impacts, the algorithm also integrates the image quality assessment block to measure the blur impact. The best matching filter sets from the pool of trained filters is selected for filtering. The mechanism for a low computational cost is also presented, resulting on 6 times less complex algorithm and a simple $l_{1}$-norm metric for class matching, without having any loss in the quality. This also paved the way for a lightweight real-time implementation of the algorithm in the resource-limited devices.

\section{REFERENCES}

Bertero, M., Boccacci, P., 1998. Introduction to Inverse Problems in Imaging. CRC Press.

Bilcu, R., Vehvilainen, M., 2008. Constrained unsharp masking for image enhancement. In: Image and Signal Processing, vol. 5099, pp. 10-19.

Crete, F., Dolmiere, T., Ladret, P., Nicolas, M., 2007. The blur effect: perception and estimation with a newno-reference perceptual blur metric. In: SPIE Conference Series Society of Photo-Optical Instrumentation Engineers. vol. 6492.

Dabov, K., Foi, A., Katkovnik, V., Egiazarian, K., 2008. Image restoration by sparse 3D transform-domaincollaborative filtering. In: SPIE Electronic Imaging. 
Daubechies, I., Defrise, M., De Mol, C., 2004. An iterative thresholding algorithm for linear inverse problems with a sparsity constraint. Comm. Pure Appl. Math. 57(11), 1413-1457.

Figueiredo, M., Nowak, R., 2003. An em algorithm for wavelet-based image restoration. IEEE Transactions on Image Processing, 12(8), 906-916.

Foi, A., Dabov, K., Katkovnik, V., Egiazarian, K., 2006. Shape-adaptive DCT for denoising and imagereconstruction. In: PROC. SPIE Electronic Imaging.

Hansen, P.C., 1998. Rank-deficient and Discrete ill-posed Problems: Numerical Aspects of Linear Inversion. Society for Industrial and Applied Mathematics.

Horiuchi, T., Watanabe, K., Tominaga, S., 2007. Adaptive filtering for color image sharpening and denoising. In: International Conference on Image Analysis and Processing Workshops. pp. 196-201.

Hradi, M., Kotera, J., Zemk, P., roubek, F., 2015. Convolutional neural networks for direct text deblurring. In: Proceedings of the British Machine Vision Conference (BMVC).

Joshi, N., Zitnick, C., Szeliski, R., Kriegman, D., 2009. Image deblurring and denoising using color priors. In: IEEE Conference on Computer Vision and Pattern Recognition. pp. 1550-1557.

Kondo, T., Kobayashi, N., Takahashi, K., Watanabe, Y., 2002. Picture converting apparatus, picture converting method, learning apparatus, learning method, and record medium, US Patent 6,414,717.

Krishnan, D., Fergus, R., 2009. Fast Image Deconvolution using Hyper-Laplacian Priors. In: Advances in Neural Information Processing Systems 22, pp. 1033-1041.

Kupyn, O., Budzan, V., Mykhailych, M., Mishkin, D., Matas, J., 2017. Deblurgan: Blind motion deblurringusing conditional adversarial networks. ArXiv e-prints.

Levin, A., Fergus, R., Durand, F., Freeman, W.T., 2007: Deconvolution using natural image priors. ACM Trans. Graphics 26(3), pp. 0-2.

Levin, A., Weiss, Y., Durand, F., Freeman, W., 2009. Understanding and evaluating blind deconvolutionalgorithms. In: IEEE Conference on Computer Vision and Pattern Recognition. pp. 1964-1971.

Ling, S., Hui, Z., de Haan, G., 2008. An overview and performance evaluation of classification-based leastsquares trained filters. IEEE Transactions on Image Processing 17(10), pp. 1772-1782.

Mainali, P., Wittebrood, R., 2015. Machine learning deconvolution filter kernels for image restoration. In: Proc. SPIE. Electronic Imaging vol. 9401, pp. 940104-13.

Nah, S., Kim, T.H., Lee, K.M., 2017. Deep multi-scale convolutional neural network for dynamic scenedeblurring. In: The IEEE Conference on Computer Vision and Pattern Recognition (CVPR).

Nam, M., Ahuja, N., 2012. Learning human preferences to sharpen images. In: International Conference onPattern Recognition. pp. 2173-2176.

Price, J., Gee, T., Tobin, K., 2001. Blur estimation in limited-control environments. In: IEEE International Conference on Acoustics, Speech, and Signal Processing. vol. 3, pp. 1669-1672.

Rieder, P., Scheffler, G., 2001. New concepts on denoising and sharpening of video signals. IEEE Transactions on Consumer Electronics 47(3), pp. 666-671.

Schmidt, U., Schelten, K., Roth, S., 2011. Bayesian deblurring with integrated noise estimation. In: IEEE Conference on Computer Vision and Pattern Recognition. pp. 2625-2632.

Schuler, C.J., Burger, H.C., Harmeling, S., Scholkopf, B., 2013. A machine learning approach for non-blindimage deconvolution. In: Proceedings of IEEE Conference on Computer Vision and Pattern Recognition. pp. 1067-1074.

Vogel, C.R., 2002. Computational Methods for Inverse Problems. Society for Industrial and Applied Mathematics.

Vonesch, C., Unser, M., 2008. A fast thresholded Landweber algorithm for wavelet-regularized multidimensional deconvolution. IEEE Transactions on Image Processing 17(4), pp. 539-549.

Xu, L., Ren, J.S., Liu, C., Jia, J., 2014. Deep convolutional neural network for image deconvolution. In: Advances in Neural Information Processing Systems 27, pp. 1790-1798.

Xu, L., Tao, X., Jia, J., 2014. Inverse kernels for fast spatial deconvolution. In: 13th European Conference on Computer Vision ECCV. pp. 33-48.

Zoran, D., Weiss, Y., 2011. From learning models of natural image patches to whole image restoration. In: IEEE International Conference on Computer Vision. pp. 479-486. 